\title{
Optimal Detection of Multiple Symbol-Slotted Random Access based Packet Transmissions
}

\author{
Steven Kisseleff, Member, IEEE and Wolfgang H. Gerstacker, Senior Member, IEEE
}

\begin{abstract}
Random access (RA) protocols are suitable for the support of massive connectivity especially in machine-type communications, such as the Internet of Things (IoT). In this context, ALOHA protocols (both slotted and unslotted) have been re-introduced as promising enablers due to a potentially high throughput when enhanced via successive interference cancellation (SIC). Meanwhile, SIC has become the most popular solution for RA detection problems.

In this work, we first point out the weaknesses of SIC and then propose a novel method based on joint detection of all randomly scheduled data packets. The proposed method is based on the joint computation of a-posteriori probabilities for all data symbols and packet localizations, such that optimal detection for RA is obtained.
\end{abstract}

Index Terms-Random access, joint detection, MAP, ALOHA

\section{INTRODUCTION}

$\mathbf{R}$ ANDOM access (RA) to medium resources has recently re-gained the attention of the research community due to the massive connectivity envisioned for both terrestrial and satellite-assisted Internet of Things (IoT) as well as beyond $5 \mathrm{G}$ communication systems [1], [2]. In such networks, the nodes may wake up at random instants of time and start their transmission without having to wait for a free slot or a grant as in traditional medium access schemes.

In many communication systems, packet-based slotted transmissions are preferred where each time slot has a fixed length corresponding to the length of a data packet and uncoordinated interference is avoided. In contrast, in unslotted RA multiple packet transmissions are allowed to overlap in time without any prior timing or frame synchronization. Such unslotted transmission offers an additional flexibility for the network design but requires highly complex detection methods since the missing synchronization imposes an additional uncertainty for the symbol detection compared to slotted RA [3]. Symbol-based slotted RA assumes a sufficiently accurate sampling phase synchronization but does not require any frame synchronization. Hence, this type of RA provides an increased flexibility compared to the packet-based slotted RA but does not entail such an extremely high computational complexity for the detection as the unslotted RA. In this context, the traditional slotted and unslotted ALOHA protocols have been enhanced with additional features such as error correction [4], replica transmissions [5] and interference cancellation [6], and spread-spectrum approaches [7].

Steven Kisseleff is with the Interdisciplinary Centre for Security, Reliability and Trust (SnT), University of Luxembourg, Luxembourg, E-mail: steven.kisseleff@uni.lu.

Wolfgang H. Gerstacker is with the Institute for Digital Communications, Friedrich-Alexander University Erlangen-Nürnberg (FAU), Erlangen, Germany, E-mail: wolfgang.gerstacker@fau.de.
The typical characteristics that are used to describe RA are medium access (MAC) load, packet length and number of users. The knowledge of these quantities at the receiver can be exploited to improve the detection reliability [8].

There are two main strategies for detecting multiple overlapping data streams: joint detection (JD, cf. [9]) and iterative processing via successive interference cancellation (SIC, cf. [6]). In particular, JD is optimal in case of continuous transmissions and known communication channels for each node. However, for discontinuous transmissions of short data packets with known length, JD according to the state of the art is suboptimal since the knowledge of the packet length is not incorporated into the detection process. In contrast, SIC based detectors incorporate all available information from the respective node in each iteration, since interference cancellation requires a complete packet detection and decoding [6], [7]. However, SIC can only operate properly if combined with packet localization $^{1}$. Hence, prior to detection a packet localization needs to be performed which is typically associated with preamble detection via correlation, cf. [7]. Due to a time-varying interference variance ${ }^{2}$, preamble correlation may not always be reliable. Intuitively, the correlation should be replaced by a maximum ratio combining in presence of noise/interference power fluctuations [12]. Furthermore, if a correlation operation is applied in the SIC receiver, such scheme is clearly suboptimal since the characteristics of the interfering signals (i.e., packet lengths, probability of transmission, etc.) are not taken into account, cf. [8].

A better strategy would be to perform a JD of all data streams by taking into account all known quantities of the underlying random processes since an optimal RA receiver should exploit all available information. As an example, JD in [9] is not fully optimal due to the multi-stage processing where the localization of the transmitted packets is performed prior to the symbol detection. In fact, none of the existing JD frameworks is optimal since either the packet localization is applied prior to the symbol detection or the symbol detection is done prior to the selection of the packet position hypothesis.

In this work, we propose a novel method of JD for symbolbased slotted RA based on concatenated Markov chains. A joint packet localization and data detection is performed by computing the a-posteriori probabilities (APPs) for all sym-

${ }^{1}$ In fact, all packet-based slotted ALOHA protocols rely on perfect frame synchronization and thus packet localization, cf. [4], [10]. Furthermore, SIC relies on the capture effect, i.e., on sufficiently separable signals with large power discrepancy [11].

${ }^{2}$ Here, we refer to the interference from the colliding transmissions which have not yet been removed by SIC, cf. [8]. 
bols of all overlapping data packets resulting in an optimal detection performance. Hence, the performance of this detector corresponds to an upper bound for any practical scheme.

This paper is organized as follows. In Section II, the system model is introduced. A novel method of packet detection is described in Section III. A performance analysis is provided in Section IV. Section V concludes the paper.

\section{System MODEL}

We assume that $N$ network links are active simultaneously in principle and each corresponding node $n$ may start transmitting its data packet with probability $p_{n}$ in the current time step. The knowledge about the transmission probability is either acquired from the nodes via measurements or available according to the system characteristics. ${ }^{3}$ Note that the distribution of idle and busy symbol intervals, which depends on the transmission probability, is beyond the scope of this work. Unlike previous works where each packet transmission is supported by the transmission of multiple replicas or segments of the code word, we focus on a single transmission per packet.

For the signal detection, we consider an observation window of $W$ symbols. The transmitted data is encoded via forward error correction (FEC) coding and linearly modulated. Each data packet from node $n$ consists of $L_{n}$ symbols and has the duration $L_{n} T$, where $T$ is the symbol interval and $L_{n} \ll W, \forall n$ holds. The number of symbols in each packet $L_{n}$ is known to the receiver, since it corresponds to the length of the employed FEC codeword. Furthermore, we assume that the transmission is impaired by additive white Gaussian noise (AWGN) with a constant known variance $\sigma_{N}^{2}$. While intersymbol interference effects are modeled as negligible, an extension of the proposed scheme to frequency-selective channels is possible in principle. ${ }^{4}$

We denote the symbols of packet $l$ of node $n$ as $a_{n, l}[m], 0 \leq$ $m<L_{n}$. We consider the use of a finite alphabet $\mathcal{A}_{n}$ with $M_{n}$ symbols $c_{n, i} \in \mathcal{A}_{n}, 1 \leq i \leq M_{n}$ for modulation. In addition, an accurate symbol level synchronization is assumed such that each node knows when the symbol intervals begin and transmits its data packets accordingly. The discrete time instant of beginning of packet $l$ of node $n$ is denoted as $\tau_{n, l}$. Note that $\tau_{n, l}$ is an integer number which corresponds to an offset relative to the first symbol of the observed sequence of symbols. Furthermore, $\tau_{n, l}$ may differ for different nodes. The packets belonging to the same node are modeled as nonoverlapping. The total data sequence transmitted by node $n$ consists of an infinite number of packets and is given by

$$
x_{n}[m]=\sum_{l=0}^{\infty} a_{n, l}\left[m-\tau_{n, l}\right], \forall n, 0 \leq m<W .
$$

The received signal $y[m]$ at the input of the detector comprises the transmit signals $x_{n}[m], \forall n$ weighted by a fixed complexvalued channel coefficient $h_{n}$ and discrete-time AWGN $w[m]$,

$$
y[m]=\sum_{n=1}^{N} h_{n} x_{n}[m]+w[m], 0 \leq m<W .
$$

\footnotetext{
${ }^{3}$ In some applications, $p_{n}$ may be related to the duty cycle of the nodes and thus is known. Otherwise, it can be estimated based on prior detections.

${ }^{4}$ For this, the state definitions in Section III.B and III.C have to be extended to include all past data symbols affecting a receive symbol.
}

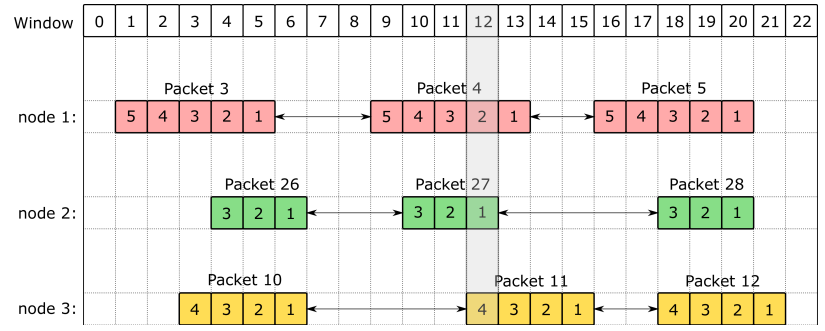

Fig. 1. Sequence of symbols received from three nodes with $L_{1}=5, L_{2}=3$ and $L_{3}=4 ; W=23$. As an example, in the 13th symbol interval we obtain $y[12]=h_{1} a_{1,4}[3]+h_{2} a_{2,27}[2]+h_{3} a_{3,11}[0]+w[12]$. Note that the packet state (in colored square) is set according to the proposed method.

In this paper, we assume ideal channel knowledge since we focus on the principle of the proposed detection algorithm. ${ }^{5}$ An example of packet reception and the composition of the received signal $y[m]$ is shown in Fig. 1. For the decoding of the messages of each of the nodes we utilize a soft-decision decoder which is based on the Viterbi algorithm (VA). As input to the VA, log-likelihood ratios (LLRs) based on APPs are needed. Thus, the optimum computation of LLRs taking into account all available signal characteristics including the packet length should be addressed. In the following, we propose such method for computation of LLRs / APPs for multiple overlapping short packet transmissions.

\section{OPTIMAL PACKET DETECTION}

\section{A. Outline of the method}

The transmission of a packet from node $n$ with parameters $h_{n}, p_{n}, L_{n}$ and $\mathcal{A}_{n}$, is modeled as a Markov process. Correspondingly, in line with general detection approaches for Markov processes, conditional probabilities characterizing the transitions between any two states of the packet are obtained and stored in a state transition matrix $\mathbf{P}$, and conditional probabilities of the observations $y[m]$ for given transitions are computed and stored in a likelihood matrix $\mathbf{R}[m]$. Then, the well-known BCJR algorithm (cf. [14]) is applied in order to determine the joint probabilities (JPs) for observing the received signal $y[m], \forall m$ and an occurrence of a given state $s[m]$. Subsequently, the APPs are determined by combining the JPs and used to calculate the LLRs for the VA. In the following, we discuss the calculation of matrices $\mathbf{P}$ and $\mathbf{R}[\mathrm{m}]$ based on specifically designed Markov chains as well as the calculation of the APPs.

\section{B. Markov chain for transmissions from a single node}

For simplicity, we start with the detection of randomly scheduled data packets from a single node, i.e., $N=1$. Since the packet length is assumed to be known at the receiver, we define a 'packet state' as a number of receive symbols to be still affected by the data packet including the current symbol. For example, once a new data packet arrives, its packet state is $L_{1}$, see Fig. 1. In the subsequent symbol intervals the packet state reduces to $L_{1}-1, L_{1}-2$, etc. A packet state ' 0 ' indicates

${ }^{5}$ In a practical application of the advocated scheme, channel estimation has to be incorporated, exploiting e.g. training sequences transmitted for initial symbol synchronization also for channel acquisition, and embedding per-survivor processing, cf. [13], in the BCJR algorithm for channel tracking. 


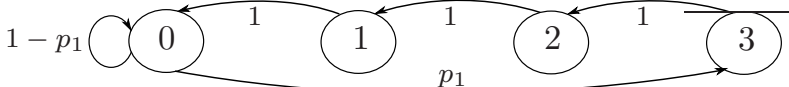

Fig. 2. Markov chain with packet states for a single node and $L_{1}=3 . p_{1}$ denotes the probability of transmission.



Fig. 3. Extended Markov chain with states comprising packet states and hypothetical transmit symbols with $N=1, L_{1}=3$, and binary modulation.

the absence of the useful signal in a particular symbol interval. An example of a corresponding Markov chain for $L_{1}=3$ is shown in Fig. 2. Here, we have only four possibilities ('0', '1', '2' and ' 3 ') for the respective packet state. The transition from state ' 0 ' into state ' 3 ' occurs with probability $p_{1}$ corresponding to the transmission of a new packet. A transition from states ' 3 ', ' 2 ' and ' 1 ' is only possible into states ' 2 ', ' 1 ' and ' 0 ', respectively. This indicates that a packet transmission will not stop before all symbols of the packet are transmitted.

In addition, we need to distinguish between the different transmitted symbols in each symbol interval of the data packet. To this end, we append a hypothetical symbol from $\mathcal{A}_{1}$ to each packet state. Hence, multiple sets of states are obtained, where each set corresponds to a particular current transmit symbol. We denote these sets as $\mathcal{S}_{c, 1}$. As an example, for a binary modulation alphabet $\mathcal{A}_{1}=\{-1,+1\}$ and a packet length $L_{1}=5,[4,-1] \in \mathcal{S}_{-1,1}$ and $[2,+1] \in \mathcal{S}_{+1,1}$ are two possible states. All valid combinations of packet states and hypothetical transmit symbols are interpreted as states of an extended Markov chain. With a given a-priori probability distribution of the symbols in the alphabet and a given transmission probability $p_{1}$, the transition probability between any two states of the extended Markov chain can be calculated. In case of equal a-priori probabilities, the transition from a Markov chain state pertaining to the packet state $1<q \leq L_{1}$ into a Markov chain state corresponding to the packet state $q-1$ occurs with a probability of $1 / M_{1}$. In Fig. 3 , the resulting extended Markov chain for $L_{1}=3$ and binary modulation is shown. Apparently, there are $1+L_{1} M_{1}=7$ states corresponding to concatenated packet states and transmit symbols: [0], $[1,+1],[2,+1],[3,+1],[1,-1],[2,-1],[3,-1]$. In addition, we show a trellis diagram according to the extended Markov chain of Fig. 3 in Fig. 4. For a transmission of two packets $[+1,-1,-1]$ and $[-1,+1,-1]$, the path through the trellis diagram is marked in red.

\section{Markov chain for transmissions from multiple nodes}

Next, we modify the above approach of state definition for random transmissions from multiple nodes.

A Markov product-chain is created resulting from combining multiple extended Markov chains pertaining to the different nodes. Each state of this product-chain corresponds to a concatenation of respective states of the individual extended

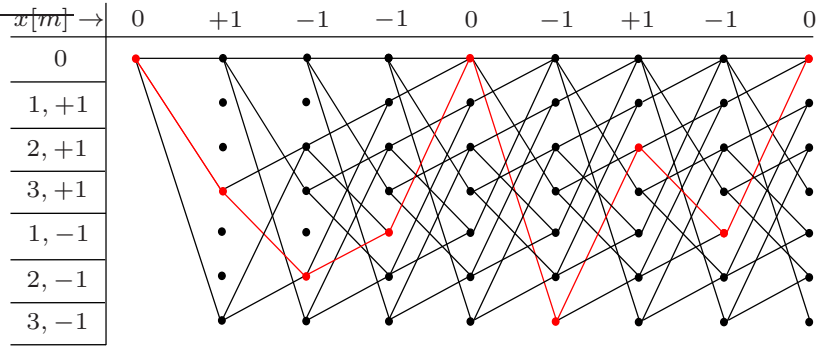

Fig. 4. Trellis diagram for a single node, $L_{1}=3$, and BPSK modulation. The path corresponding to a transmission of two packets $[+1,-1,-1]$ and $[-1$, $+1,-1]$ is marked in red.

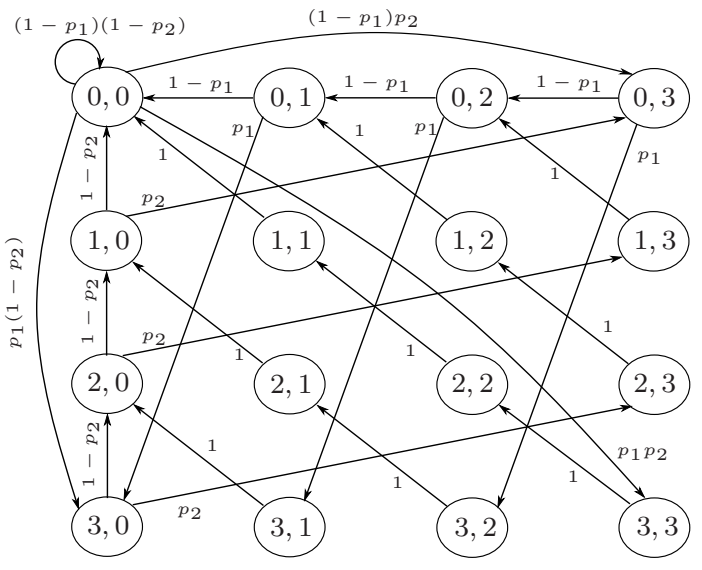

Fig. 5. Markov chain of packet states for two nodes and $L_{1}=L_{2}=3$.

chains. For instance, $([3,-1],[5,+1],[0],[1,+1])$ is a possible state for $N=4$ and $L_{n}=5, \forall n$. For the clarity of exposition, we show an example of a Markov chain for two nodes without the transmit symbols, i.e., only the packet states are depicted, see Fig. 5. The state of the product-chain in time $m$ is denoted as $s_{p}[m]$, and $\tilde{a}_{n}\left(s_{p}[m]\right)$ represents the symbol transmitted by the $n$th node when the product-chain is in state $s_{p}[m]$. The transition probabilities between the states of the product-chain are calculated via multiplication of the transition probabilities between respective states of the individual extended chains, since the transmissions from different nodes are statistically independent. It should be noted that for dependent transmissions the transition probabilities should be calculated differently.

\section{Joint probabilities}

APPs can be computed using the BCJR algorithm which utilizes matrices $\mathbf{P}$ and $\mathbf{R}[m]$, where $\mathbf{P}$ contains the transition probabilities between any two states of the Markov product-chain and $\mathbf{R}[m]$ comprises the conditional pdf values $\operatorname{pdf}\left(y[m] \mid s_{p}[m]\right)$ related to the transmission channel for time instant $m, 0 \leq m<W$. In the presence of AWGN, the conditional pdf $\operatorname{pdf}\left(y[m] \mid s_{p}[m]\right)$ is given by

$$
\operatorname{pdf}\left(y[m] \mid s_{p}[m]\right)=\frac{\exp \left(-\frac{\left|y[m]-\sum_{n} h_{n} \tilde{a}_{n}\left(s_{p}[m]\right)\right|^{2}}{\sigma_{N}^{2}}\right)}{\pi \sigma_{N}^{2}} .
$$

All pdf values $\operatorname{pdf}\left(y[m] \mid s_{p}[m]\right)$ are determined and stored in matrix $\mathbf{R}[m]$ after reception of the whole sequence $y[m], 0 \leq$ $m<W$ denoted as $y[\cdot]$ in the following.

The BCJR algorithm applies a forward and a backward recursion based on the two matrices and determines JPs 
$\lambda\left(s_{p}[m]\right)=\operatorname{Pr}\left(y[\cdot], s_{p}[m]\right)$ for each state of the Markov product-chain, cf. [14]. Next, we utilize these JPs in order to detect the data streams and calculate the APPs.

Obviously, this approach for calculating the JPs can be applied for an arbitrary number of nodes, various symbol constellations, and packet lengths. The main challenge in this context is the computational complexity which is related to the number of Markov product-chain states given by $\prod_{n=1}^{N}\left(1+L_{n} M_{n}\right)$. According to [15], the required number of multiplications per information bit in the BCJR algorithm is proportional to the number of states. Hence, we obtain the asymptotic complexity $\mathcal{O}\left(W \prod_{n=1}^{N} L_{n} M_{n}\right)$. Furthermore, the total number of elements in matrices $\mathbf{R}[\mathrm{m}]$ indicates a high consumption of memory given by $\mathcal{O}\left(W \prod_{n=1}^{N} L_{n}^{2} M_{n}^{2}\right)$. However, the number of states associated with JD can be reduced using the methods described in [16]. In addition, reduced-state BCJR algorithms can be employed in practice, cf. [17], which entail a trade-off between performance and complexity. Alternatively, the proposed scheme can be incorporated into a SIC based detection scheme, considering two or three users simultaneously in each iteration step of SIC for a performance improvement.

\section{E. Detection of data streams}

In order to obtain a valid solution which corresponds to actual data packets rather than arbitrarily arranged symbols, it is necessary to locate the packets within the received sequence of symbols. ${ }^{6}$ For this, we generate all possible hypotheses $\mathcal{H}\left(\mathcal{T}_{k}\right), \forall k$ for the positions of packets, where $k$ is the hypothesis index. Each hypothesis is described by a set $\mathcal{T}_{k}$, which contains $\tau_{n, l}, \forall n, l$. Given $\mathcal{T}_{k}$, it is possible to distinguish between valid and invalid JPs, since $\mathcal{T}_{k}$ confines the packet state of each node in each symbol interval. We propose to consider the sum of all JPs belonging to a given hypothesis $\mathcal{H}\left(\mathcal{T}_{k}\right)$ irrespective of the transmit symbols as hypothesis selection criterion. A full search over all hypotheses is performed with respect to this criterion and the best hypothesis $\mathcal{H}\left(\mathcal{T}_{\max }\right)$ is chosen for the further processing.

For the symbol detection, the JPs that simultaneously pertain to the same transmit symbol of a node and to the selected hypothesis (i.e., only time instants within the selected packet locations are considered) are summed up to obtain APPs which are used to determine the LLRs,

$$
\operatorname{LLR}_{n}[m, i]=\log \left(\sum_{s_{p}[m] \in \mathcal{X}_{1}^{n, i}} \lambda\left(s_{p}[m]\right) / \sum_{s_{p}[m] \in \mathcal{X}_{0}^{n, i}} \lambda\left(s_{p}[m]\right)\right),
$$

where $\mathcal{X}_{1}^{n, i}$ and $\mathcal{X}_{0}^{n, i}$ denote the set of states which pertain to 1 and 0 , respectively, for the $i$ th bit of the $m$ th data symbol. ${ }^{7}$

\section{F. Pilot symbols}

The use of known symbols (pilots) can substantially enhance the detection performance. Here, the individual Markov chains are adjusted such that states pertaining to invalid

\footnotetext{
${ }^{6}$ Note that this sequential processing does not lead to a suboptimality of the proposed method according to the arguments in Section I, since all available information has already been incorporated in the calculation of the JPs.

${ }^{7}$ This notation follows the convention for the description of bit-interleaved coded modulation [18].
}

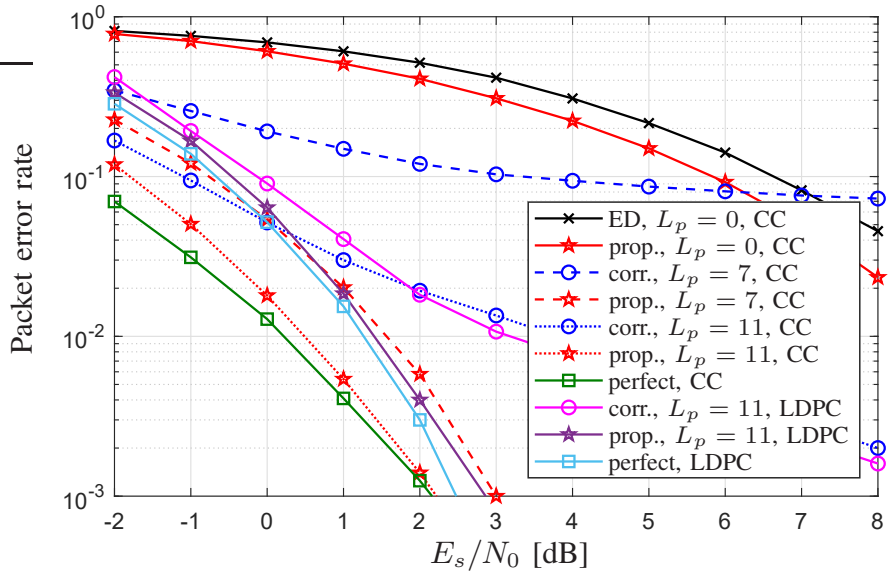

Fig. 6. Decoding performance for $L_{p} \in\{0,7,11\}$ and a single node.

symbols at the dedicated pilot positions are removed. As an example, consider $L_{p}=2$ pilot symbols $[+1,+1]$ at positions $q_{1}=1$ and $q_{2}=2$ of the packet with $L_{1}=4$. In this case, the extended Markov chain would consist of the following states: $[0],[1,+1],[1,-1],[\mathbf{2}, \mathbf{+ 1}],[\mathbf{3},+\mathbf{1}],[4,+1],[4,-1]$. Although any pilot sequence would improve the performance, we have observed that sequences with low cross-correlation perform best.

\section{NUMERICAL RESULTS}

For our simulations, we assume that the data is encoded with a convolutional code (CC) with code rate $1 / 3$, constraint length 7 , and generator polynomials [155 123 137] in octal notation (no puncturing), and binary modulation is employed, $\mathcal{A}_{1}=\{-1,+1\}$. The probability of transmission is set to $p_{1}=$ 0.1 . The channel magnitudes for all nodes are set to one while the channel phases are randomly selected but known to the receiver. The received energy per symbol and the power spectral density of the AWGN is denoted as $E_{s}$ and $N_{0}$, respectively. Furthermore, we employ Barker sequences of length $L_{p} \in\{7,11\}$ as pilot sequences in order to enable an accurate packet detection, given by $[+1,+1,+1,-1,-1,+1,-1]$ and $[+1,+1,+1,-1,-1,-1,+1,-1,-1,+1,-1]$, respectively. We consider the detection of a single randomly scheduled packet per node in our simulations. The results obtained using the proposed method are compared with the traditional SIC method, where the packet detection is accomplished via correlation of the received signal with the pilot sequence, and with a genie-aided detector, which has a perfect knowledge of the packet position and thus corresponds to an upper performance bound for the symbol detection. The performance is evaluated in terms of packet error rate (PER) determined by averaging over $10^{4}$ transmitted packets.

In Fig. 6, we show the impact of the pilot sequence on the detection performance for packet transmissions with $L_{1}=$ $30+L_{p}$ from a single node. Since correlation is not feasible with $L_{p}=0$, we employ an energy detector (ED) for this case. We observe that the proposed method substantially outperforms the correlation method for all relevant values of $L_{p}$. The reason is that the information about the packet structure is well incorporated in the proposed detection scheme while only the preamble is taken into account in correlation based detection. 


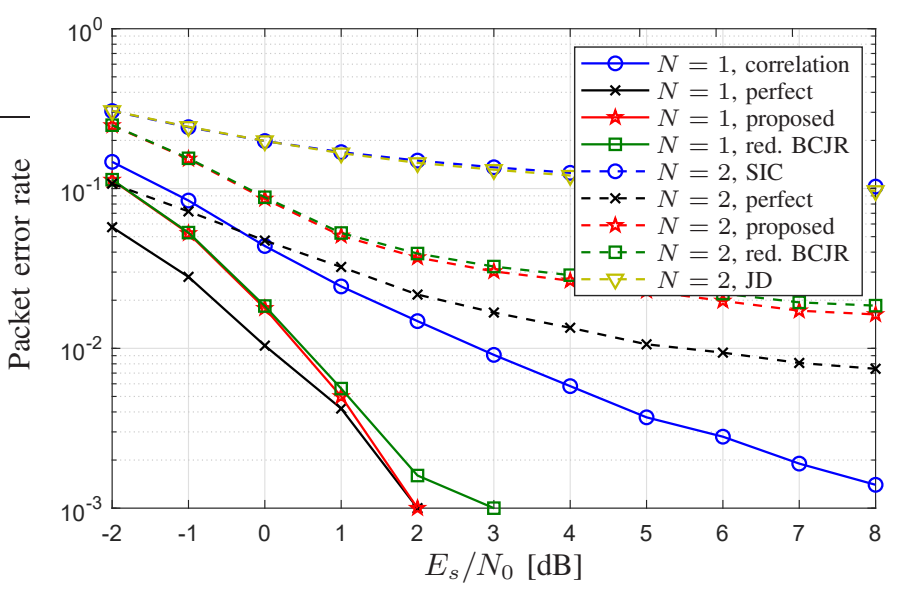

Fig. 7. Decoding performance for $L_{p}=11$ and $N=\{1,2\}$.

Furthermore, for $L_{p}=0$, the ED is outperformed by the new method since the latter utilizes not only the information about the received energy during packet transmission but also about the absence of the useful signal in other symbol intervals. For $L_{p}=11$, the PER of the proposed method is already very close to the upper performance bound. In order to demonstrate the usefulness of the proposed scheme also for other FEC codes and other code rates, we show the decoding performance for an LDPC code of rate $1 / 2$. Here, a MacKay-Neal based LDPC matrix has been employed, and a log-domain sumproduct algorithm has been used for decoding. We can observe similar gains of the proposed method compared to correlation based detection ( $L_{p}=11$ in both cases) and an only slight degradation compared to the upper bound. Due to a higher code rate of the adopted LDPC code compared to the used convolutional code, the performance of the LDPC code is somewhat inferior in the considered range of packet error rates.

In Fig. 7, we show results obtained for transmissions from one and two nodes, respectively, with $L_{1}=L_{2}=23$ and $L_{p}=11$. We restrict ourselves to a small number of nodes due to a very high computational complexity and high memory requirements otherwise. We observe a substantial performance degradation with two nodes compared to transmissions from a single node. Nevertheless, the proposed method clearly outperforms SIC based detection. Moreover, for both methods an error floor occurs in the PER curve due to the remaining uncertainty related to the mutual interference between the nodes. Still, it is possible to reach PERs in the order of $2 \cdot 10^{-2}$ with the proposed method which is typically sufficient in the majority of scenarios related to massive machine-type communication systems. Furthermore, we observe that the PER with the proposed method and two nodes is only two times higher than the theoretical upper bound given by the genie detector. Such performance close to the upper bound is in agreement with the fact that the LLRs are computed in an optimum way in the proposed method. Note that a multi-stage JD scheme, similar to [9], has been implemented for comparison, which shows a slight PER reduction compared to SIC. In addition, we show results obtained using a reduced-state BCJR algorithm, where the complexity has been drastically reduced to a total of 110 multiplications per BCJR execution. We observe only a slight performance degradation with increasing
$E_{s} / N_{0}$ compared to the full-state BCJR algorithm.

\section{CONCLUSION}

In this paper, a novel detection method for multiple random access based packet transmissions has been proposed. Our approach makes use of a joint MAP detection of symbols from multiple nodes accounting for the individual packet lengths. The proposed method is optimal with respect to the total symbol error rate of all nodes. Furthermore, it noticeably outperforms SIC. Since the complexity of the proposed method is very high, only a few overlapping data streams can be supported in a practical application. In the presence of a large number of nodes, suboptimal methods might be preferred and could be developed in future work.

\section{REFERENCES}

[1] L. Atzori, A. Iera, and G. Morabito, "The internet of things: A survey," Computer Networks, vol. 54, no. 15, pp. 2787-2805, 2010.

[2] O. Kodheli et al., "Satellite Communications in the New Space Era: A Survey and Future Challenges," July 2020, arXiv:2002.08811.

[3] R. De Gaudenzi, O. del Río Herrero, S. Cioni, and A. Mengali, "Random access versus multiple access," in Multiple Access Techniques for $5 G$ Wireless Networks and Beyond. Springer, 2019, pp. 535-584.

[4] E. Paolini, G. Liva, and M. Chiani, "Coded slotted ALOHA: A graphbased method for uncoordinated multiple access," IEEE Transactions on Information Theory, vol. 61, no. 12, pp. 6815-6832, 2015.

[5] E. Casini, R. De Gaudenzi, and O. Del Rio Herrero, "Contention Resolution Diversity Slotted ALOHA (CRDSA): An Enhanced Random Access Scheme for Satellite Access Packet Networks," IEEE Transactions on Wireless Communications, vol. 6, no. 4, pp. 1408-1419, 2007.

[6] Y. Tadokoro, H. Okada, T. Yamazato, A. Katayama, and A. Ogawa, "A new packet detection scheme in CDMA unslotted ALOHA system with successive interference cancellation," in Proc. of IEEE Global Communications Conference, 2001, pp. 3173-3177.

[7] O. Del Rio Herrero and R. De Gaudenzi, "High Efficiency Satellite Multiple Access Scheme for Machine-to-Machine Communications," IEEE Transactions on Aerospace and Electronic Systems, vol. 48, no. 4, pp. 2961-2989, 2012.

[8] S. Kisseleff, J. Kneissl, G. Kilian, and W. H. Gerstacker, "Efficient detectors for telegram splitting-based transmission in low power wide area networks with bursty interference," IEEE Transactions on Communications, vol. 68, no. 12, pp. 7687-7701, 2020.

[9] P. Kota and C. Schlegel, "A wireless packet multiple access method exploiting joint detection," in Proc. of IEEE International Conference on Communications (ICC), vol. 4, 2003, pp. 2985-2989.

[10] C. Stefanovic, P. Popovski, and D. Vukobratovic, "Frameless ALOHA Protocol for Wireless Networks," IEEE Communications Letters, vol. 16, no. 12, pp. 2087-2090, 2012.

[11] E. Balevi, F. T. Al Rabee, and R. D. Gitlin, "ALOHA-NOMA for massive machine-to-machine IoT communication," in Proc. of IEEE International Conference on Communications (ICC), 2018, pp. 1-5.

[12] S. Verdu, Multiuser Detection. Cambridge University Press, 1998.

[13] R. Raheli, A. Polydoros, and C.-K. Tzou, "Per-Survivor Processing: a General Approach to MLSE in Uncertain Environments," IEEE Transactions on Communications, vol. 43, no. 2/3/4, pp. 354-364, Feb./March/April 1995.

[14] L. Bahl, J. Cocke, F. Jelinek, and J. Raviv, "Optimal decoding of linear codes for minimizing symbol error rate," IEEE Trans. on Information Theory, vol. 20, no. 2, pp. 284-287, Mar 1974.

[15] S. Wang and F. Patenaude, "A systematic approach to modified BCJR MAP algorithms for convolutional codes," EURASIP Journal on Advances in Signal Processing, vol. 2006, no. 1, p. 095360, 2006.

[16] R. E. Learned, A. S. Willsky, and D. M. Boroson, "Low complexity optimal joint detection for oversaturated multiple access communications," IEEE Transactions on Signal Processing, vol. 45, no. 1, pp. 113-123, 1997.

[17] D. Fertonani, A. Barbieri, and G. Colavolpe, "Reduced-complexity BCJR algorithm for turbo equalization," IEEE Transactions on Communications, vol. 55, no. 12, pp. 2279-2287, 2007.

[18] G. Caire, G. Taricco, and E. Biglieri, "Bit-interleaved coded modulation," IEEE Transactions on Information Theory, vol. 44, no. 3, pp. 927-946, 1998. 